\title{
Inhibition of Addictive Behaviors by Peer-Group Effect in a Conditional Delayed Reward Task
}

\author{
Takashi Sato
}

\begin{abstract}
In the recent years, the excessive reliance on games and the internet has escalated to pathological levels, especially in adolescents, and is interfering with their lives. This issue can be regarded as one of the delayed reward tasks. In this study, we propose a conditional delayed reward task in which players can get a small reward by continuing to play the game instead of studying, but eventually receive large negative rewards. In order to investigate the cause and countermeasures of this problem, we adopt a reinforcement learning model and focus on the peer-group effect (PGE) in which positive or negative influences given by the group to individuals is one of the ways of inhibiting the problem. Our simulation results showed that in case of the group without the PGE, individuals with a small gamma value (one of the parameters of the reinforcement learning model suggested to correspond to the amount of serotonin in the brain), continued to play the game and almost attained a state of addiction. We also found that, in the condition with the PGE, players who were game dependent in the absence of the PGE, chose studying over gaming.
\end{abstract}

Keywords: Internet gaming disorder; Inhibition of addictive behaviors; Peer-group effect; Conditional delayed reward task; Reinforcement learning

\section{INTRODUCTION}

$\mathrm{I}^{\mathrm{n}}$ $\mathrm{n}$ the recent years, the problem of relying too much on games and the internet has escalated to pathological levels, especially in adolescents, and is interfering with their lives. In 2013, the American Psychiatric Association (APA) claimed that Internet Gaming Disorder (IGD) became a condition for further study in the DSM-5 [1], and the World Health Organization (WHO) has officially recognized IGD as an international disease and in 2018 added the IGD to the latest edition of the ICD-11 [2]. The WHO defined IGD as "a pattern of gaming behavior characterized by impaired control over gaming, increasing priority given to gaming over other activities to the extent that gaming takes precedence over other interests and daily activities, and continuation or escalation of gaming despite the occurrence of negative consequences [3]."

The relationship between the brain and IGD has been investigated. The functional magnetic resonance imaging studies indicated that adolescents with IGD showed lower white matter in several brain regions that are involved in decision-making, behavioral inhibition, emotional regulation, had decreased functional connectivity of prefrontal cortex striatal circuits, and increased risk-taking [4]. Other recent studies, which resemble the IGD study, suggest that the internet addiction disorder (IAD) is associated with structural abnormalities in the brain gray matter, and these brain abnormalities probably contribute to chronic dysfunction [5].

As ways to treat people with IGD and IAD, some methodologies are adopted such as cognitive behavioral therapies [6-7] and medication therapies [8-9]. The Kurihama Medical and Addiction Center, which is a special hospital in Japan, uses a mixture of cognitive behavioral therapy, social skills development, and treatment programs emphasizing physical activity [10]. Higuchi who is the director of the center has claimed that "in some ways addiction to gaming is harder to treat than addiction to alcohol or drugs because the internet is everywhere [11]." As indicated above, the IGD and the IAD are fairly recent to officially recognized as international diseases. Therefore, the medical treatments for the IGD and the IAD are under study yet. In particular, methods for preventing and inhibiting the IGD and the

Corresponding Author: Takashi Sato. Department of Media Information Engineering, National Institute of Technology, Okinawa College, 905, Henoko, Nago, Okinawa, 905-2192, Japan, Tel: +81-980-55-4179, Fax: +81980-55-4012, E-mail: stakashi@ okinawa-ct.ac.jp 
IAD for people before affected with the IGD and the IAD are remained to be established.

In this study, we consider that people are constantly influencing each other's behaviors. Most people have smartphones with data connections, which can be connected to the internet and used to play games. Therefore, an individual not engaged in these activities, may still be influenced by others around him/her and within sight. This situation is known to exert a "PeerGroup Effect (PGE) [12-14]," influencing all people in a group mutually. We think of PGE as a way to potentially inhibit addictive behaviors such as IGD and IAD. PGE can be expressed as a proverb "He who touches pitch will be defiled." That is to say; if people watch many individuals who behave a certain way, they will be affected by these individuals to behave similarly. This, however, means that PGE can have both positive and negative influences on people.

The purpose of this study is to clarify whether PGE can actually inhibit addictive behaviors. In order to achieve this, we proposed a conditional delayed reward task (CDRT) as a model of situations that can be expressed as addictive behavior, like IGD, and adopted a model of reinforcement learning. The reason why this situation can be regarded as a kind of delayed reward task is that game players gain positive rewards that can be quite enjoyable in the short term, but may eventually result in negative rewards, such as bad grades.

\section{CONDITIONAL DELAYED REWARD TASK}

\section{A. Situation of the Task Selecting Two Types of}

\section{Behaviors: Playing a Game or Studying}

We are constantly making choices and in most cases, we try to make choices that will benefit us, through estimations based on our previous experiences and information obtained in the past. There are situations in which selecting the best choice can be very difficult, but there are also ones in where correct choices can be estimated easily. For instance, in schools, there are many students who are unable to make the appropriate choice of studying over the more harmful alternatives, such as playing games, despite the knowledge of regularly scheduled weekly tests.

In this study, we modeled a task as a CDRT to students. The students were regarded as the agents, and there were three states: s0-s2. State s0 was the initial state. The state incremented each time all agents acted. They got a small, positive, immediate reward by playing a game (indicated by $\mathrm{G}$ ) or a small negative immediate reward by studying (indicated by $\mathrm{S}$ ) at each state.
However, a large negative delayed reward was given to them after continued game play, and a large positive delayed reward was earned after continued study. Therefore, in order to avoid getting a big negative delayed reward, they had to continue to select studying with small negative immediate rewards. The CDRT expressed by a state transition diagram is depicted in Fig. 1. As seen in Fig. 1, we defined four types of conditions for the method of giving the delayed rewards. Four types of delayed rewards were given to the players after they were added to the immediate reward given at state s2,

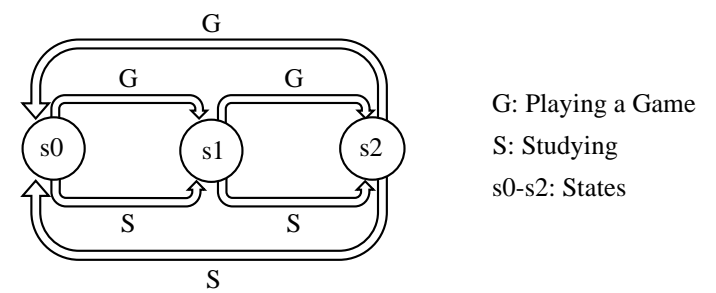

\footnotetext{
$<$ Immediate Reward>

- A small positive reward is given when $\mathrm{G}$ is selected at each state.

- A small negative reward is given when $\mathrm{S}$ is selected at each state.

$<$ Conditional Delayed Reward>

After the action at state $\mathrm{s} 2$ :

- A large positive reward is added if $\mathrm{G}$ has not been selected and

$\mathrm{S}$ is selected three times. $(\mathrm{G}=0, \mathrm{~S}=3)$

- A small positive reward is added if $\mathrm{G}$ is selected once and

$\mathrm{S}$ is selected twice. $(\mathrm{G}=1, \mathrm{~S}=2)$

- A small negative reward is added if $\mathrm{G}$ is selected twice and

$\mathrm{S}$ is selected once. $(\mathrm{G}=2, \mathrm{~S}=1)$

- A large negative reward is added if $\mathrm{G}$ is selected three times and

$\mathrm{S}$ has not been selected. $(\mathrm{G}=3, \mathrm{~S}=0)$
}

Fig. 1 A state transition diagram of the conditional delayed reward task.

according to defined conditions.

In the CDRT, actions that kept an agent playing a game, despite the big penalties that were eventually received were regarded as addictive behaviors. We confirmed whether PGE could inhibit addictive behaviors by using the CDRT.

\section{B. Reinforcement Learning Agents as Players of the} Task

We adopted reinforcement learning agents as the players of the task and Q-learning [15] as the learning method. Q-learning [15] is one of the reinforcement learning methods by trial and error. In accordance with the following (1), the agents with the Q-learning renew the Q-values representing the worth of the agents' actions 
for each state of the environment and obtain more appropriate behaviors.

$$
\begin{aligned}
& Q(s(t), a(t)):=Q(s(t), a(t))+\alpha[r(t+1) \\
& \left.\quad+\gamma \max _{a} Q(s(t+1), a)-Q(s(t), a(t))\right],
\end{aligned}
$$

where $a(t)$ is an action conducted at time $t, Q(s(t), a(t))$ is a Q-value for an action conducted under a state $s$ at time $t, \alpha$ is the learning rate, $r(t+1)$ is a reward given from the environment when conducting an action under a state $s$, and $\gamma$ is the discount rate. The softmax policy [15] was adopted as the method of selecting actions.

\section{Peer-Group Effect in the Task}

PGE [12-14] is an influence exerted by the members in a group, mutually and strongly affected by the majority. For example, it is known that a phenomenon emerges in which the achievement of low-performing students can be elevated by a positive influence from the high-ranking students in a class. PGE, however, not only has positive impacts as presented above, but also negative ones, and peer pressure can result in undesirable influence. We define PGE below:

$P_{a(t)}^{P G E}=\frac{1}{2}\left(P_{a(t)}+F_{a(t-1)}\right)$,

$F_{a(t)}=\frac{n_{a(t)}}{N}$,

where $P_{a(t)}^{P G E}$ is the probability with the PGE to select an action at time $t, P_{a(t)}$ is a probability without the PGE to select an action determined by the softmax policy at time $t, F_{a(t)}$ is the rate of an action selected by all agents at time $t, n_{a(t)}$ is the number of an action selected by all agents at time $t$, and $N$ is the number of agents. The range of each important value is $0 \leq P^{P G E}, P, F \leq 1$.

As understood from (2) and (3), action choice probabilities influenced by the PGE depend on the rate of an action selected by all agents. Therefore, even if an agent has a possibility of selecting action $\mathrm{S}$ with high probability, the agent selects the other action $(\mathrm{G})$ if there were many agents who selected action $\mathrm{G}$, and vice versa.

Conversely, a situation without PGE can be regarded as a circumstance in which the agents are not mutually affected by anyone, such as when staying in his/her private room.

\section{SIMULATION EXPERIMETNS}

\section{A. Settings of the Simulations}

The parameters used in the simulation experiments are as follows: The small positive reward given to an agent who selected action $\mathrm{G}$ at every state was +0.1 , and the small negative reward given to an agent who selected action $\mathrm{S}$ at every state was -0.1 .

After the agent gained the immediate reward at state s2, the delayed reward, according to the conditions, was added to the immediate reward. As illustrated in Fig. 1, we set four types of conditions: a large positive reward added to the immediate reward at state s2 was +10.0 when the agent selected his/her action $\mathrm{S}$ three times, a small positive reward was added (+5.0) when the agent selected his/her action $S$ two times, a small negative reward was added (-5.0) when the agent selected his/her action $S$ once, and a large negative reward (-10.0) was added when the agent never selected action $\mathrm{S}$ from s0 to s2, namely, he/she only selected action G.

The number of agents in a group was 121, because there were mixed agents with all combinations of the following parameters: 11 types of the learning rate $\alpha=$ $\{0.01,0.1,0.2,0.3,0.4,0.5,0.6,0.7,0.8,0.9,0.99\}$ and 11 types of the discount rate $\gamma=\{0.01,0.1,0.2,0.3,0.4$, $0.5,0.6,0.7,0.8,0.9,0.99\}$. We conducted the simulation experiments until 100,000 steps every twenty different kinds of random seeds.

\section{B. Results of the Simulations}

We investigated the impact of PGE on addictive behaviors by comparing the CDRT without PGE to the one with PGE.

First, we confirmed selection probabilities of action $\mathrm{G}$ at each state, for all 121 types, to investigate the effects of their parameters on selecting action $\mathrm{G}$ without PGE. Fig. 2 (a)-(c) show examples of the dynamics of selection probabilities of action $\mathrm{G}$ at the three states, $\mathrm{s} 0$ s2, for each of the 121 types of agents, respectively. As can be seen in Fig. 2 (a), there are three clusters: the first one is the group of agents who can learn to avoid selecting action $\mathrm{G}$, the second one is the group of agents for which the selection probabilities of action $G$ range from 0.2 to 0.6 , and the third one is the group of agents who cannot learn to inhibit selecting action G, i.e., these selection probabilities are 1.0. As can be seen in Fig. 2 (b), there are also some agents for whom the selection probabilities of action $\mathrm{G}$ are 1.0 and many agents with a variety of selection probabilities between 0.0 and 0.6. Fig. 2 (c), however, illustrates that all agents can learn to avoid selecting action $\mathrm{G}$. 


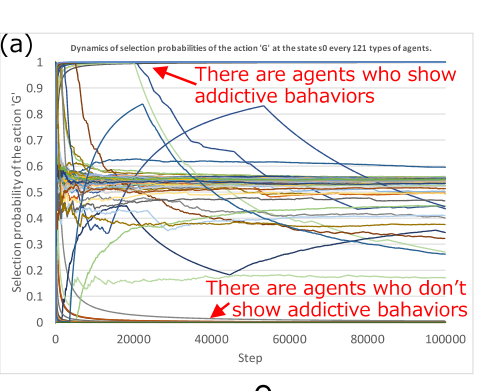

s0

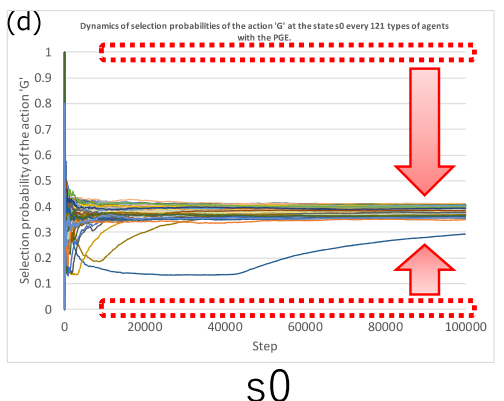

\section{Without PGE}

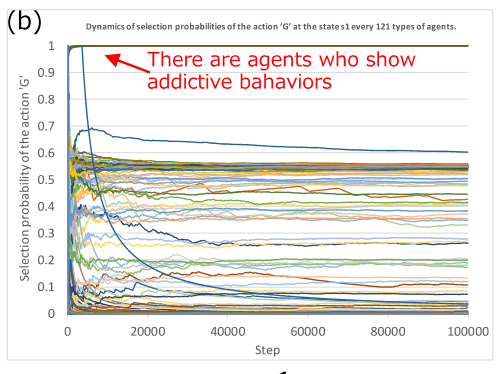

s1

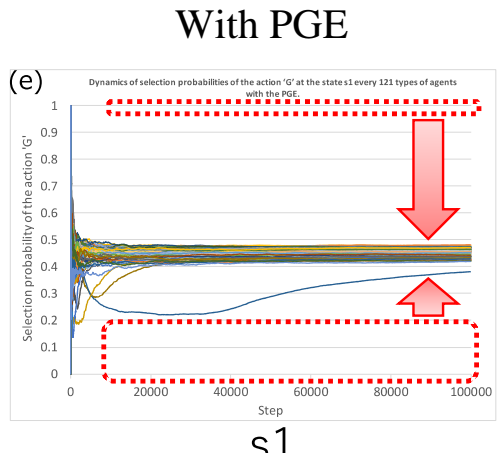

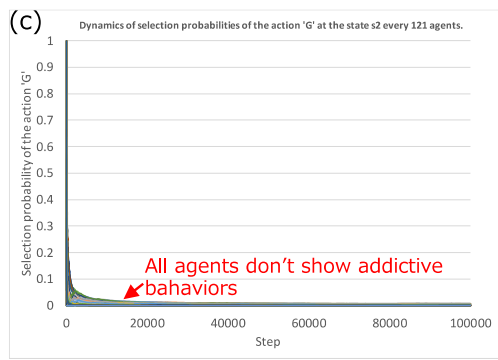

s2

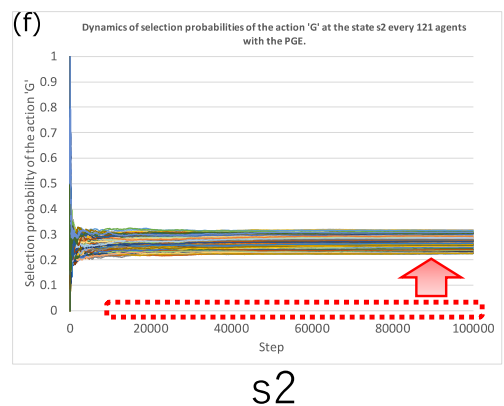

Fig. 2 Examples of dynamics of selection probabilities of action $\mathrm{G}$ at each state for all 121 types of agents without PGE (a)-(c), and with PGE (d)-(e).

On the other hand, as can be seen in Fig. 2 (d)-(f), all selection probabilities range from 0.2 to 0.5 at each state. It is of particular importance that there were no agents with addictive behaviors for which the selection probabilities for action $\mathrm{G}$ were 1.0. The results shown in Fig. 2 (d)-(f) suggest that addictive behaviors could be inhibited by PGE; however, no agents learned to completely avoid selecting action G. The minority agents, who were supposed to select action $\mathrm{S}$, got into the mood of playing the game when there were many agents who selected action G. This was thought to be a result of the negative impact of the PGE on the agents.

Similar results to the ones shown in Fig. 2 (a)-(f) were confirmed in other simulations with different random seeds.

Next, we illustrate the ensemble average rates of action $\mathrm{G}$ selected at each state for all 121 types of agents, for the last 1000 steps. Fig. 3 (a)-(c) are heatmaps showing the rates without PGE, and (d)-(f) depict the rates with PGE. These ensemble average results were calculated by averaging the results of the simulations independently conducted with 20 different random seeds. The agents with a high discount rate $\gamma$ basically appreciate the action where a positive reward may be gained in the future. Therefore, even if such agents continuously get small negative rewards as the immediate reward, as long as they earn a large positive reward later, they can easily select the action that contributes to getting the large positive reward.

From Fig. 3 (a) and (b), which show results without PGE, we found that the ensemble average rates of action $\mathrm{G}$ selected at state $\mathrm{s} 0$ and $\mathrm{s} 1$ decreased as the learning rates of the agents increased, only when the discount rates were high to some extent. Note that even if the agents have high discount rates, they increased the rates of action $\mathrm{G}$ with extremely low learning rates, such as 0.01 . Furthermore, most agents with discount rates more than 0.9 increased the rates of action $G$ inversely. The reason that most agents could learn to select action $\mathrm{S}$ at state $\mathrm{s} 2$, as shown in Fig. 3 (c), is that s2 is a state providing either the largest positive or negative reward to the agents in accordance with the conditions. The agents who cannot learn to select action S continuously learn to avoid action $G$ at state s2 since the largest negative rewards are given at that state. In contrast, the agents who can continue selecting action $\mathrm{S}$ reinforce 


\section{Without PGE}

(a)

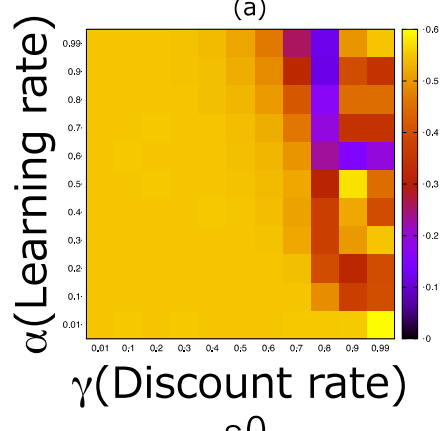

s0

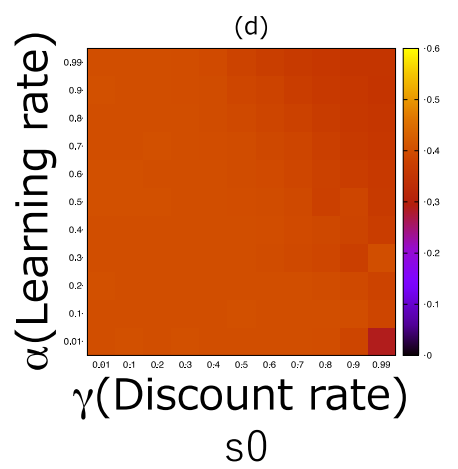

(b)

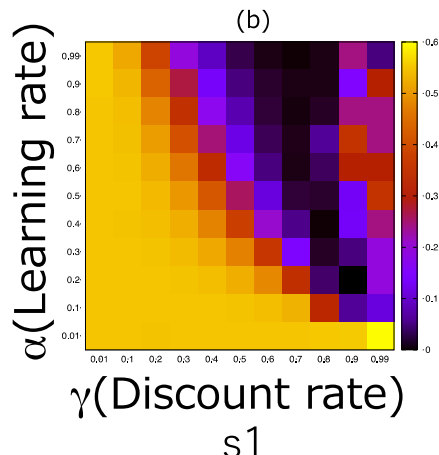

With PGE

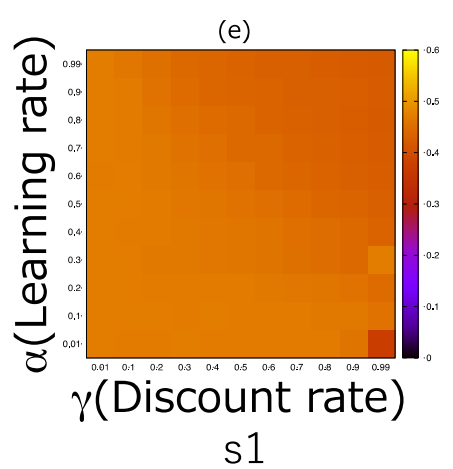

(c)
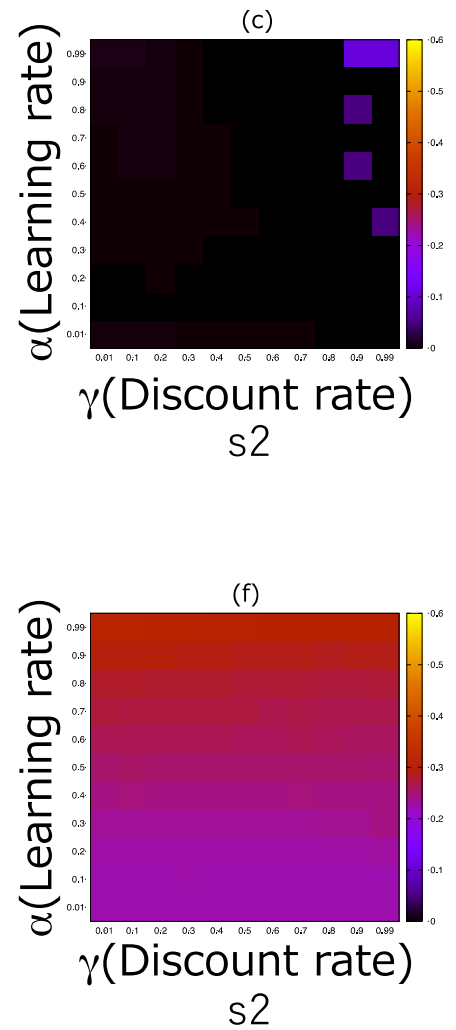

Fig. 3 Ensemble average rates of action G selected at each state for all 121 types of agents without PGE (a)-(c), and with PGE (d)-(f), during the last 1000 steps.

selecting action $\mathrm{S}$ at state $\mathrm{s} 2$ since the largest positive rewards are given at that state.

Fig. 3 (d)-(f) show the ensemble average rates of action $\mathrm{G}$ selected at each state for all 121 types of agents with PGE. An important difference between Fig. 3 (a)(c) and (d)-(f) is that in the latter cases, there are rarely effects from the discount and learning rates except for at state s2. In Fig. 3 (d)-(f), a common feature is that there are rarely effects of the discount rate, but the ensemble average selection rate of action $G$ is extremely small decayed as both the discount and the learning rates are increased. In contrast to the results without PGE, most of the smallest selection rates of action $\mathrm{G}$ at state $\mathrm{s} 0$ and $\mathrm{s} 1$ are earned when the discount rate is 0.99 and the learning rate is 0.01 (the rates are 0.3 and 0.37 at $\mathrm{s} 0$ and $\mathrm{s} 1$, respectively). On the other hand, in state s2, the ensemble average selection rates of action $\mathrm{G}$ depend on the learning rate. As can be seen in Fig 3 (f), the learning rate 0.5 is a boundary, and the ensemble average selection rate of action $G$ decreased to 0.23 as the learning rate decreased from 0.5 to 0.0 . The ensemble average selection rate of action $\mathrm{G}$, however, increased to 0.31 as the learning rate increased from 0.5 to 1.0. That is to say, in contrast to the situation without PGE, in the one with PGE, it is necessary for the agents to have low learning rates to inhibit addictive behaviors.

Finally, we show a comparative result between the higher-than-average selection rates of action $\mathrm{G}$ without PGE and with PGE. As can be seen in Fig. 4, except for state s2, the higher-than-average selection rates of action $\mathrm{G}$ with PGE are less than the ones without PGE. This suggests that the effect of inhibiting addictive behaviors by positive PGE becomes smaller every time the state is transited, and in state s2, the effect is finally reversed, and the attraction to addictive behaviors by negative PGE becomes stronger. 


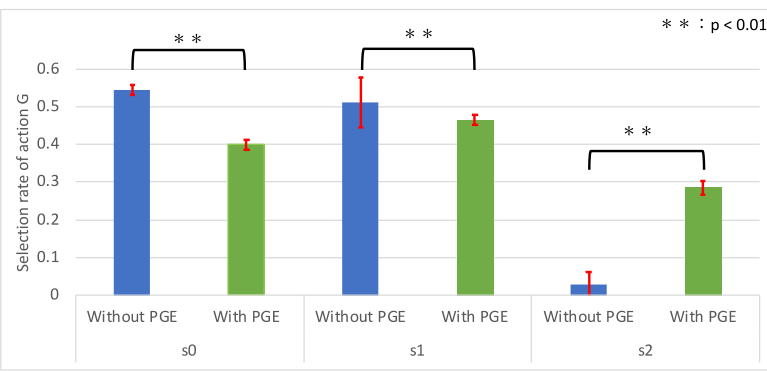

Fig. 4 Comparison between the higher-than-average selection rates of action $\mathrm{G}$ without PGE (blue bar) and with PGE (green bar).

\section{DISCUSSIONS}

We discuss the results showed in our simulations from the viewpoint of the relations between parameters of the reinforcement learning and our brains. In this paper, we focused on the learning rate $\alpha$ and the discount rate $\gamma$ as important parameters of reinforcement learning.

The learning rate $\alpha$ was a parameter to determine the amount of updating the action value. If the learning rate $\alpha$ was large, the agent drastically changed his/her action value according to the reward received. However, the agent with a small learning rate $\alpha$ hardly changed his/her action value. In our brain, acetylcholine, one of the neurotransmitters, is suggested to play an important role as a gate signal that decides whether to keep past memories or exchange it for new ones [16]. The learning rate $\alpha$ can be said to have corresponded to acetylcholine.

It is also known that, compared to adult brains, adolescent brains have high plasticity that dynamically changes their brains, including synapses, neurons, and axons [17-19]. Konrad et al. [20] suggested that there is a possibility that typical adolescent behavior patterns, including risk-taking, are caused by the high plasticity of the adolescent brain. Based on these suggestions, we presumed that the agents with high learning rates, who could be regarded as adolescents with high plasticity, easily show addictive behaviors. In our simulation results without PGE, the agents with high learning rates, however, showed relatively appropriate behaviors in only cases that they had a high discount rate. This result suggests that in case of a situation without influences from other individuals, people can learn to avoid selecting addictive behaviors by drastically changing their action values in accordance with the large negative reward. In contrast, agents with PGE, like people in a classroom, influence each other and can learn to avoid extreme behaviors, but most of the agents become individuals who select inappropriate behaviors by receiving negative impact from the PGE, even though the big delayed reward is given.

The discount rate $\gamma$ is a parameter to determine which of immediate and delayed rewards emphasize. As the brain learns, related to the discount rate, Okamoto et al. [21] showed that serotonin plays an important role in controlling a time scale parameter for reward prediction. Therefore, the discount rate $\gamma$ can be regarded as representing serotonin. In fact, our simulation results without PGE corresponded to the results conducted by Okamoto et al., and the agents with low discount rates, relatively easily selected the action that received an immediate positive reward. Furthermore, Miyazaki et al. [22-23] clarified that, in a delayed reward task using rats, increasing flow rate of serotonin and activating the serotonin nervous system was related to waiting for the delayed reward. The experimental results by Miyazaki et al. can be interpreted that there are the two modes of the discount rates in which a large $\gamma$ gives high praise to delayed reward, but a small $\gamma$ does not do so, and the two modes are switched by the amount of serotonin. Our simulation results showed that PGE can inhibit addictive behaviors to some extent, but the complete avoidance of addictive behaviors was not seen. In the situations with PGE, where the agents could not completely learn to avoid addictive behavior, we thought that it was necessary for them to not only increase their discount rate, namely their amount of serotonin, but also producing only positive PGE.

\section{CONCLUSION}

In this paper, we investigated whether the PeerGroup Effect (PGE) could have an influence of inhibiting addictive behaviors. For this, we proposed a conditional delayed reward task (CDRT) as a model for situations that can be expressed as addictive behaviors and adopted a model of reinforcement learning agents as players in the CDRT.

From our simulation results, comparing the CDRT without PGE and with PGE, we found that PGE has an impact to inhibit addictive behaviors to some extent. However, we confirmed that agents who could learn to completely avoid inappropriate actions did not emerge because of the negative influences of the PGE.

In order to completely inhibit the addictive behaviors in a space like a classroom that can have a PGE, we conclude that the approach of increasing the amount of serotonin, which contributes to gain delayed rewards, is not enough, and a method to produce only positive PGE is also needed such as some controlling a portion of the students by intervention by teachers. 


\section{ACKNOWLEDGEMENTS}

The author thanks Akira Masumi, Takeshi Konno and Takashi Hashimoto for their fruitful discussions. This work was supported by JSPS KAKENHI Grant Number JP16H06397.

\section{REFERENCES}

[1] The American Psychiatric Association (Eds.), Diagnostic and Statistical Manual of Mental Disorders, Fifth Edition (DSM-5), 2013.

[2] The World Health Organization (Eds.), International Statistical Classification of Diseases and Related Health Problems, Eleventh Edition (ICD-11), 2018.

[3] The World Health Organization (Eds.), "Gaming disorder," Online Q\&A on the official website of the WHO, 2018. https://www.who.int/features/qa/gaming-disorder/en/

[4] A.M. Weinstein, "An update overview on brain imaging studies of internet gaming disorder," Frontiers in Psychiatry, vol.8, issue 185 , https://doi.org/10.3389/fpsyt.2017.00185

[5] K. Yuan, et al., "Microstructure abnormalities in adolescents with internet addiction disorder," PLoS one, vol.6, issue 6, e20708, 2011.

[6] L. Huanhuan, W. Su, "The role of cognitive distortion in online game addiction among Chinese adolescents," Children and Youth Services Review, vol.33, issue 9, pp.1468-1475, 2013.

[7] M.W.R. Stevens, D.L. King, D, Dorstyn, P.H. Delfabbro, "Cognitive-behavioral therapy for Internet gaming disorder: A systematic review and meta-analysis," Clinical Psychology \& Psychotherapy, vol.26, issue 2, pp.191-203, 2018.

[8] J. Song, J.H. Park, D.H. Han, et al., "Comparative study of the effects of bupropion and escitalopram on Internet gaming disorder," Psychiatry and Clinical Neurosciences, vol.70, issue 11, pp.527-535, 2016.

[9] J.H. Park, Y. Lee, J.H. Sohn, D.H. Han, "Effectiveness of atomoxetine and methylphenidate for problematic online gaming in adolescents with attention deficit hyperactivity disorder," vol.31, issue 6, pp.427-432, 2016.

[10] The Kurihama Medical and Addiction Center, Introduction of the treatment of internet addiction and research (TIAR) (in Japanese). https://kurihama.hosp.go.jp/hospital/section/internet.html

[11] G. Humphreys, "Sharpening the focus on gaming disorder: the definition of gaming disorder is an important first step in developing a public health response to a new problem," Bulletin of the World Health Organization, vol.97, issue 6, pp: 382-383, 2019.

[12] R. Arnott, J.G. Rowse, "Peer group effects and educational attainment," Journal of Public Economics, vol.32, issue 3, pp.287-305, 1987.

[13] W.N. Evans, W.E. Oates, R.M. Schwab, "Measuring peer group effects: a study of teenage behavior," The Journal of Political Economy, vol.100, No.5, pp.966-991, 1992.

[14] S. You, "Peer influence and adolescents' school engagement," Procedia - Social and Behavioral Sciences, vol.29, pp.829-835, 2011.
[15] R.S. Sutton, A.G. Barto, Reinforcement Learning: An Introduction, MIT Press, 1998.

[16] M.E. Hasselmo, "Neuromodulation: acetylcholine and memory consolidation," Trends in Cognitive Sciences, vol.3, issue 9, pp.351-349, 1999.

[17] W.B. Gan, E. Kwon, G. Feng, J.R. Sanes, J.W. Lichtman, "Synaptic dynamism measured over minutes to months: age-dependent decline in an autonomic ganglion," Nature Neuroscience, vol.6, issue 9, pp.956-960, 2003.

[18] J. He, F.T. Crews, "Neurogenesis decreases during brain maturation from adolescence to adulthood," Pharmacology Biochemistry and Behavior, vol.86, pp.327-333, 2007.

[19] Y. Zuo, P. Chang, A. Lin, W.B. Gan, "Development of long-term dendritic spine stability in diverse regions of cerebral cortex," Neuron, vol.46, issue 2, pp.181-189, 2005.

[20] K. Konrad, C. Firk, P.J. Uhlhaas, "Brain development during adolescence: neuroscientific insights into this developmental period," Deutsches Ärzteblatt international 2013, vol.110, issue 25, pp. 425-431, 2013.

[21] Y. Okamoto, et al., "Effects of serotonin on delay discounting for rewards -an application for understanding of pathophysiology in psychatric disorders- (in Japanese)," Psychiatria et Neurologia Japonica, vol.114, issue 2, pp.108-114, 2012.

[22] K. Miyazaki, K.W. Miyazaki, K. Doya, "Activation of dorsal raphe serotonin neurons underlies waiting for delayed rewards," Journal of Neuroscience, vol.31, issue 2, pp.469-479, 2011.

[23] K.W. Miyazaki, K. Miyazaki, K. Doya, "Activation of the central serotonergic system in response to delayed but not omitted rewards," European Journal of Neuroscience, vol.33, issue 1, pp.153-160, 2011. 